\title{
Entwicklung einer österreichischen Forschungsagenda für onkologische Pflege
}

\author{
Eine Delphi-Studie
}

\author{
Silvia Raphaelis ${ }^{1}$ Sabine Köck-Hódi ${ }^{1}$ Hanna Mayer ${ }^{1}$
}

Eingegangen: 30. September 2016 / Angenommen: 6. Juli 2017 / Online publiziert: 7. August 2017

(C) Der/die Autor(en) 2017. Dieser Artikel ist eine Open-Access-Publikation.

\section{Zusammenfassung}

Hintergrund Pflegeforschung ist eine wesentliche Komponente bei der Verbesserung der Versorgung onkologisch erkrankter Menschen. Da der Forschungsbedarf hoch ist und gleichzeitig die Forschungsmittel begrenzt sind, bietet eine Forschungsagenda eine grundlegende Orientierung, um vorrangige Themen zu bearbeiten.

Ziel Ziel dieser Arbeit war die Entwicklung einer österreichischen Agenda für onkologische Pflegeforschung, die sowohl an die internationale Diskussion um Forschungsprioritäten als auch an die nationale Pflegepraxis anschließt. Methoden Im ersten Schritt wurde eine Literaturstudie zu pflegerelevanten onkologischen Forschungsprioritäten durchgeführt. Die hierbei identifizierten Forschungsprioritäten wurden mittels einer Delphi-Befragung von ExpertInnen aus Österreich hinsichtlich ihrer Relevanz hierzulande bewertet, und in weiterer Folge wurde eine Forschungsagenda mit entsprechender nationaler Schwerpunktsetzung erstellt.

Ergebnisse Insgesamt konnten 11 Artikel in die Literatursynthese eingeschlossen werden. In der ersten Befragungsrunde haben 18, in der zweiten 15 und in der dritten Befragungsrunde 13 ExpertInnen teilgenommen. Die finale Forschungsagenda ist aus 9 übergeordneten Forschungsbereichen (z. B. Entwicklung und Testung von Interventionen) und 43 zugehörigen Forschungsprioritäten (z. B. Interventionen zum Symptommanagement) zusammengesetzt.

Silvia Raphaelis

silvia.mona.raphaelis@univie.ac.at

1 Institut für Pflegewissenschaft, Fakultät für Sozialwissenschaften, Universität Wien, Alser Straße 23/12, 1080 Wien, Österreich
Schlussfolgerungen Die Forschungsagenda bietet eine wesentliche Orientierung für die Entwicklung der onkologischen Pflegeforschung in Österreich. Sie ist ein erster wichtiger Schritt, auf dem weiter aufgebaut werden muss, um eine national gültige Forschungsagenda mit hoher Repräsentativität der Forschungsbedarfe in Österreich zur Verfügung stellen zu können.

Schlüsselwörter Onkologische Pflege . Forschungsagenda $\cdot$ Literaturreview $\cdot$ Delphi-Studie Pflegeforschung

\section{Development of an Austrian research agenda for nursing in oncology}

A Delphi study

\begin{abstract}
Background Nursing research is an essential component to improve the care of oncology patients. A research agenda provides a principle orientation to balance high needs for research and limited resources in the prioritization of topics. Aim This research aims to develop an Austrian research agenda for oncology nursing which is linked to the international discussion about research priorities as well as to the national needs of nursing practice.

Method In a first step, a literature study on research priorities relevant to nursing of oncology patients was conducted. The identified research priorities were appraised concerning their national relevance within a Delphi study by Austrian oncology nurses and a research agenda with national key points was subsequently worked out.

Results In total 11 publications were included in the literature synthesis, 18 oncology nurses participated in the first Delphi round, 15 in the second and 13 in the last round.
\end{abstract}


The research agenda in its final version is comprised of 9 main research areas (e.g. development and evaluation of interventions) containing 43 associated research priorities (e. g. interventions for symptom management) in total. Conclusion The research agenda provides a principle orientation to guide the development of oncological nursing research in Austria. It must be regarded as the first important step, which needs to be developed further to make a national valid research agenda with high representativeness of research needs available.

Keywords Oncology nursing - Research priorities · Review $\cdot$ Delphi technique $\cdot$ Nursing research

\section{Hintergrund}

Das inhaltliche Spektrum der Pflegewissenschaft umfasst ein weites Gebiet - von der Gesundheitsförderung bis zur palliativen Pflege am Lebensende. Da diesem großen Forschungsgebiet begrenzte finanzielle und personelle Ressourcen gegenüberstehen, stellt sich die Frage, welche Evidenz prioritär durch Forschung generiert werden soll. Diese Frage kann mithilfe einer Forschungsagenda geklärt werden. Denn diese enthält eine Liste an Themen (Forschungsprioritäten), auf die aktuell in der Forschung fokussiert werden soll. Dies trägt nicht nur dazu bei, dass Forschungsfragen und verfügbare Mittel dort platziert werden, wo der größte Bedarf besteht, sondern auch, dass Forschungsaktivitäten zu wichtigen Themen systematisch strukturiert, koordiniert und bearbeitet werden $[1,2]$. In Ländern wie den USA, Kanada, Großbritannien, Norwegen und Australien werden prioritäre Forschungsthemen von unterschiedlichen Fachgruppen wie etwa dem Royal College of Nursing schon seit den 1970er-Jahren in staatlich unterstützten Projekten politisch abgestimmt entwickelt [1, 3, 4]. Neben Agenden, die sich auf die gesamte Pflegeforschung beziehen, bestehen solche für spezifische Bereiche, wie etwa für die Psychiatrie, die Geriatrie oder Onkologie $[1,3]$. So gibt es für die onkologische Pflege eigene Kapitel in allgemeinen Forschungsagenden, wie in der Swiss Research Agenda for Nursing [1], aber es sind auch gesonderte Agenden verfügbar, wie jene der Oncology Nursing Society [5].

Im onkologischen Bereich sind Forschungsprioritäten aufgrund der weiten Verbreitung onkologischer Erkrankungen, der komplexen pflegerischen Praxis und der vielfältigen Probleme und Bedürfnisse der Betroffenen, aus denen sich ebenso vielfältige Themen für die Forschung ergeben, relevant. So betreffen onkologische Erkrankungen alle Regionen und sozioökonomischen Gruppen, die aufgrund der wachsenden und älter werdenden Bevölkerung als auch der steigenden Überlebensraten weiterhin an Bedeutung gewin- nen $[6,7]$. Hier stellt sich etwa die Frage, wie PatientInnen und Angehörige mit geringem Einkommen, Migrationshintergrund oder hohem Alter besser unterstützt werden können [5]. Da die gesundheitliche Versorgung zunehmend ambulant und häuslich erfolgt [8], sind sowohl die Pflegepraxis (z. B. Unterstützung des Selbst- und Alltagsmanagements durch Beratung) [9] als auch die Betroffenen (z. B. Selbstmanagement krankheits- und therapiebedingter Symptome sowie von Spät- und Langzeitfolgen) mit neuen Herausforderungen konfrontiert [10]. Die Erarbeitung entsprechender Programme zur bestmöglichen Förderung des Selbstmanagements der PatientInnen stellt hierbei etwa ein Thema für die onkologische Pflegeforschung dar [5].

Im deutschsprachigen Raum wurde bisher lediglich für die Schweiz eine Forschungsagenda für onkologische Pflege entwickelt [2]. Betrachtet man die Situation in Österreich, so werden pflegerische Studien hier zwar zunehmend durchgeführt, diese sind allerdings wenig aufeinander abgestimmt und beziehen sich schwerpunktmäßig auf klinische Fragestellungen (z. B. Symptombelastung und -management) [11, 12]. Andere Bereiche wie etwa die Versorgungs- oder Grundlagenforschung zur Entwicklung von Praxiskonzepten und theoretischen Modellen wurden bisher kaum etabliert. Demnach würde hierzulande die Ausrichtung der Forschung an einer konkreten Agenda dazu beitragen, diese gezielter zu steuern und dringende Anliegen zur Bearbeitung zu bringen. Allerdings stellt sich die Frage, inwieweit international diskutierte Forschungsprioritäten auf den österreichischen Kontext übertragen werden können, bzw. ob für die österreichische Pflegepraxis bestimmte Themen im Speziellen relevant sind. So zählt Österreich beispielsweise zu jenen Ländern, in denen neue Krebsmedikamente äußerst früh, rasch und breitflächig eingesetzt werden [13]. Dadurch müssen sich Onkologiepflegende ebenso rasch darauf einstellen, die neuen Nebenwirkungen der neuen Krebsmedikamente zu erkennen und zu behandeln [14]. Zudem weist Österreich in der onkologischen Versorgung eine ausgeprägte Zentrums- und Schwerpunktbildung auf, die sich v.a. auf die Landeshauptstädte Wien, Graz und Innsbruck konzentriert [15]. Demnach kann nur einem Teil der Bevölkerung eine wohnortnahe, spezialisierte onkologische Versorgung angeboten werden. Unabhängig davon weist die psychoonkologische und palliative Versorgung beträchtliche Defizite auf [16]. Da Österreich im europäischen Vergleich bei der Fünfjahresüberlebensrate, die in den europäischen Ländern $40-64 \%$ beträgt, mit $61 \%$ an vierter Stelle [17] liegt, besteht hierzulande außerdem ein überdurchschnittlich hoher Bedarf für die Versorgung von ,cancer survivors“. 


\section{Ziel}

Das Ziel dieser Studie ist die Entwicklung einer österreichischen Forschungsagenda für onkologische Pflege, die sowohl mit der internationalen Diskussion um Forschungsprioritäten vernetzt als auch auf die spezifische Situation der klinischen Pflegepraxis in Österreich ausgerichtet ist.

\section{Methode}

Für die Entwicklung der Forschungsagenda wurden internationale Forschungsprioritäten über eine Literaturrecherche identifiziert und anschließend eine Delphi-Befragung mit nationalen ExpertInnen durchgeführt, um zu überprüfen, inwieweit diese für die onkologische Pflegeforschung in Österreich Priorität haben.

\section{Literaturstudie}

Für die Literaturstudie wurde in methodischer Hinsicht an die schweizerische Forschungsagenda von Shaha et al. [2] angeknüpft, da hier Literatur über prioritäre Forschungsthemen aus den Jahren 1995-2006 bereits umfassend recherchiert, bewertet, analysiert und zusammengefasst wurde.

Ausgehend vom Ende des Recherchezeitraums bei Shaha et al. [2] wurden deutsch- oder englischsprachige Artikel ab 2006 zu einer explizit aus Forschenden-, Pflegenden-, PatientInnen- oder Angehörigenperspektive formulierten Forschungsagenda für den Erwachsenenbereich eingeschlossen. Dabei wurden alle Forschungsagenden berücksichtigt, die durch einen systematischen Prozess (z. B. Delphi-Studie, Review) für die Onkologieforschung an sich (Pflegeforschung neben anderen Disziplinen), die onkologische Pflegeforschung oder ihre Teilbereiche (z. B. kolorektale Onkologiepflege) erstellt wurden. Studien derselben Entwicklungsgruppe, die in Vorbereitung auf eine Forschungsagenda durchgeführt wurden und deren Ergebnisse in diese integriert wurden, wurden ausgeschlossen.

Die Literaturrecherche wurde im Juli 2013 in den Datenbanken PubMed und CINAHL mit einer Kombination aus Medical Subject Headings und freien Suchbegriffen (Tab. 1) durchgeführt und im Mai 2016 aktualisiert. Zudem wurden die Webseiten onkologischer Fachgesellschaften (z. B. Oncology Nursing Society, Onkologiepflege Schweiz) und die Referenzlisten eingeschlossener Studien durchgesehen. Nach der Entfernung von doppelten Treffern wurden die Titel oder Abstracts der gefundenen Studien und darauf folgend die Volltextversion aller verbliebenen Treffer anhand der Auswahlkriterien selektiert. Anschließend wurden die eingeschlossenen Forschungsagenden in Analogie zu Shaha et al. [2] bezüglich ihrer Fähigkeit, Prioritäten der Pflegeforschung zu identifizieren (Level 1: niedrigste
Tab. 1 Suchstrategie für die elektronische Datenbanksuche

\begin{tabular}{ll}
\hline 1 & $\begin{array}{l}\text { Research Priorities OR Research Agenda OR Health Priori- } \\
\text { ties (MeSH) }\end{array}$ \\
2 & $\begin{array}{l}\text { Oncolog* OR Cancer OR Neoplasms (MeSH) } \\
3\end{array}$ \\
4 & Oncologic Nursing (MeSH) \\
5 & Nursing Research (MeSH) OR Clinical Nursing Research \\
6 & AND 4 \\
7 & OR 5 \\
8 & 1 AND 6 6 mit dem Filter: Publikationen ab 2006 \\
\hline
\end{tabular}

${ }^{a}$ Medical Subject Heading

Fähigkeit einer Forschungsagenda, Prioritäten der Pflegeforschung zu identifizieren, bis Level 5: höchste Fähigkeit einer Forschungsagenda, Prioritäten der Pflegeforschung zu identifizieren), eingeschätzt [18].

Aus allen eingeschlossenen Forschungsagenden wurden generelle Charakteristiken (z. B. Herkunftsland, Fachbereich, Studiendesign für Entwicklung) sowie die darin beschriebenen Forschungsprioritäten extrahiert. Die extrahierten Forschungsprioritäten wurden in Anlehnung an die Kategorien von Burnette et al. [19], die auch bei Shaha et al. [2] verwendet wurden, 9 übergeordneten Forschungsbereichen zugeordnet: „Krankheits- und Therapieerfahrungen der PatientInnen“, „Entwicklung von Assessmentinstrumenten“, „Entwicklung und Testung von Interventionen“, „PatientInnenedukation“, „spezifische PatientInnengruppen“, „Cancer Survivors und Survivorship“, „Angehörige/ Familien von Menschen mit Krebserkrankungen“, „ethische und rechtliche Aspekte“ sowie „Gesundheitswesen und Entwicklung der onkologischen Pflege“. Extrahierte Forschungsprioritäten mit überschneidenden Perspektiven bezüglich mehrerer Forschungsbereiche wurden in Einzelaspekte zerlegt und demgemäß den jeweiligen Forschungsbereichen zugeordnet. Zusätzlich wurden die Forschungsprioritäten von Shaha et al. [2] in die letztendliche Literatursynthese mitaufgenommen. Die gesamte Literaturrecherche, -auswahl und -synthese wurden durch die Erstautorin durchgeführt, von der Zweitautorin begleitet und mit der Letztautorin in einem diskursiven Prozess abgeschlossen.

\section{Delphi-Studie}

Aus den literaturbasierten Forschungsprioritäten wurde ein Fragebogen für eine Delphi-Studie entwickelt, welche die zentralen Merkmale der anonymen ExpertInnenBefragung, wiederholte Befragungsrunden, eine Rückmeldung der Zwischenergebnisse sowie die Ermittlung einer statistischen Gruppenmeinung beinhaltet [20].

$\mathrm{Da}$ in Österreich keine Onkologiepflegenden in der direkten Forschung tätig sind, wurden als ExpertInnen Onko- 
logiepflegende auf einem fortgeschrittenen Level (Masteroder Bachelorabschluss bzw. onkologische Weiterbildung oder große Expertise in der Pflegepraxis aufgrund leitender Funktion oder mehrjähriger Berufserfahrung) definiert. Diese wurden über die Arbeitsgemeinschaft hämato-onkologischer Pflegepersonen in Österreich (AHOP) und die Onkologiefachgruppe des Allgemeinen Krankenhauses der Stadt Wien über eine Kontaktperson der jeweiligen Institutionen rekrutiert und hierbei schriftlich über das Vorhaben und den Umgang mit den Daten informiert.

Nach Vorliegen des ,informed consent" wurde der Fragebogen in elektronischer Form an die ExpertInnen übermittelt, der entsprechend den Literaturergebnissen 40 potenzielle Forschungsprioritäten als Items, die auf einer 4-stufigen Likert-Skala (wichtig, eher wichtig/unwichtig, unwichtig) bewertet werden konnten, enthielt. Zudem wurden die Teilnehmenden um soziodemografische Daten (z. B. Alter, Geschlecht) gebeten.

Als statistischer Übereinstimmungsgrad zwischen den ExpertInnen wurde ein Konsens von mindestens $90 \%$ hinsichtlich der Wichtigkeit (wichtig/eher wichtig) jeder Forschungspriorität vorab definiert. Die Befragung wurde 3-mal mit dem Ziel der Konsensfindung wiederholt. In Runde 1 konnten die Teilnehmenden zudem für Österreich relevante Forschungsprioritäten ergänzen. In Runde 2 wurden nur jene Items, bei denen in der Vorrunde kein Konsens erzielt wurde oder die ergänzt wurden, einbezogen. In Runde 3 wurden ebenfalls nur jene Items einbezogen, die in der Vorrunde weniger als $90 \%$ Übereinstimmung erhielten. Diese konnten als ,eher wichtig“ oder ,eher unwichtig“ bewertet werden und wurden bei der Analyse der Gesamtergebnisse als ,eher wichtige“ Forschungsprioritäten ergänzt, wenn ein Konsens von mindestens $51 \%$ vorlag. Die Daten wurden mittels deskriptiver Statistik (Häufigkeiten, Median, Mittelwert und Standardabweichung) ausgewertet. Dabei fiel der Anteil fehlender Werte über alle Befragungsrunden hinweg sehr gering aus (1,81\% mit maximal 2 fehlenden Antworten pro Item), wobei eine genauere Prüfung darauf hindeutete, dass manche Items zufällig und manche systematisch nicht bewertet wurden (z. B. Items mit 2 fehlenden Werten, zu denen die jeweiligen ExpertInnen vermutlich keine Meinung hatten). Da zufällig fehlende Werte in der statistischen Analyse ignoriert werden können [21] und ansonsten der Konsens jener Personen ermittelt werden sollte, die auch eine Meinung zu einem bestimmten Forschungsthema hatten, wurden fehlende Werte nicht miteinbezogen. Die ExpertInnen wurden in jeder Befragungsrunde einmal daran erinnert, den Fragebogen zu retournieren. Nach jeder Befragungsrunde erhielten die ExpertInnen eine anonyme Zusammenfassung der Ergebnisse, die den Konsens der Teilnehmenden (\%) bezüglich jeder in der jeweiligen Runde bewerteten Forschungspriorität sowie den Median, den Mittelwert und die Standardabweichung bezüglich deren Wichtigkeit enthielt.
Abb. 1 Flowchart zur Studienauswahl

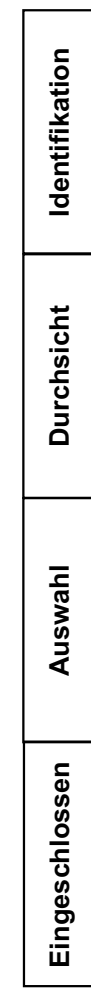

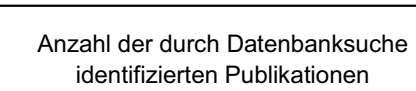

identifizierten Publikationen $(n=94)$

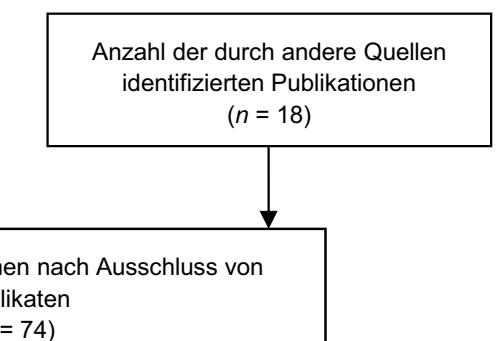

Anzahl der Publikationen nac
Duplikaten
$(n=74)$
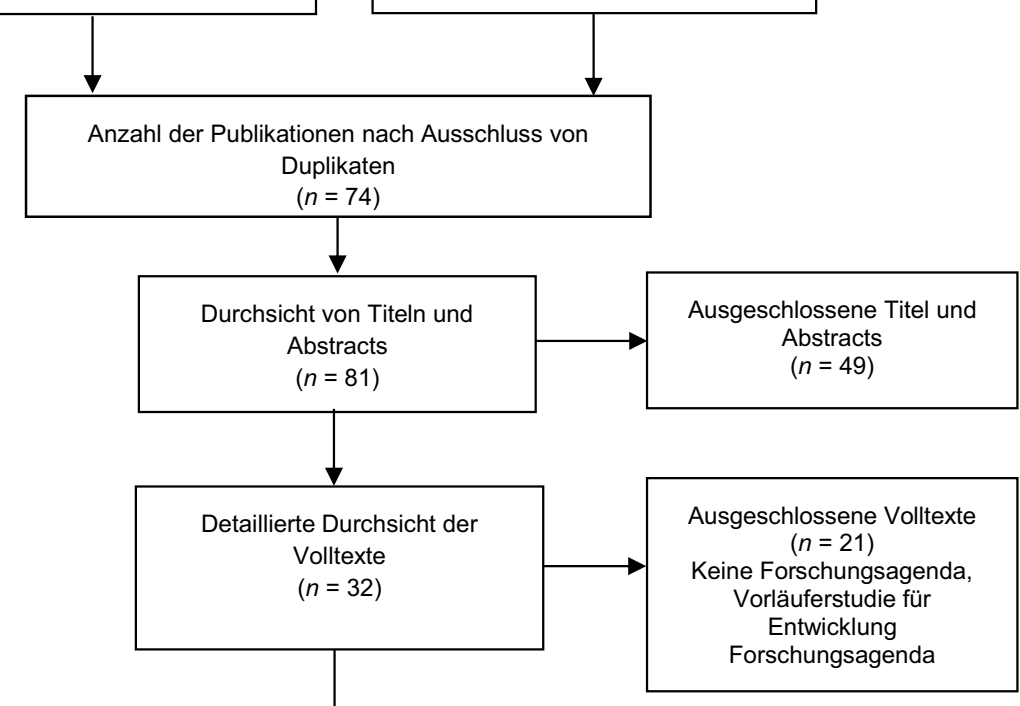

Eingeschlossen in die

Literaturübersicht $(n=11)$ 


\section{Ergebnisse}

\section{Literaturstudie}

Die Literaturrecherche führte zu insgesamt 112 Treffern, von denen 11 Publikationen bzw. Forschungsagenden in die Literatursynthese eingeschlossen wurden (Abb. 1).

Die Forschungsagenden wurden mehrheitlich für Europa [22] bzw. europäische Länder [2, 23-26] formuliert, basieren vornehmlich auf einer Literatursynthese und/oder einer quantitativen Befragung [2, 5, 22, 24-29], beziehen sich großteils auf die onkologische Pflegeforschung [2, 5, 22, 26, 29] oder ihre Teildisziplinen (z. B. Hämatoonkologie, kolorektale Onkologie, Onkogeriatrie, „cancer survivors“)
$[24,25,28,30]$ und weisen überwiegend eine hohe $[2,5$, 22, 26, 29, 30] und mittlere [24, 25, 28] Fähigkeit auf, Prioritäten der Pflegeforschung zu identifizieren (Tab. 2).

Die Literaturergebnisse wurden zu insgesamt 40 Forschungsprioritäten in 9 übergeordneten Forschungsbereichen zusammengefasst. Die Forschungsprioritäten aus dem Update [5, 28] konnten bereits bestehenden Forschungsprioritäten zugeordnet werden. Dem Forschungsbereich „Entwicklung und Testung von Interventionen“ konnten die meisten, den Bereichen „Entwicklung von Assessmentinstrumenten“ und „PatientInnenedukation“ die wenigsten Forschungsprioritäten, zugeteilt werden. Über mehrere Forschungsbereiche hinweg spielten die Themen Auswirkungen der Erkrankung und Therapie, Bedürfnisse der

Tab. 2 Charakteristiken eingeschlossener Studien

\begin{tabular}{|c|c|c|c|c|}
\hline AutorIn & Land & Bereich & Design & Level $^{\mathrm{a}}$ \\
\hline Berger et al. 2009 [29] & USA & Onkologische Pflege & $\begin{array}{l}\text { Survey mit ONS-Mitgliedern }(n=713) \text {, } \\
\text { Literatursynthese, Konsensbildung in } \\
\text { ONS }\end{array}$ & 5 \\
\hline Corner et al. 2007 [23] & UK & Onkologieforschung & $\begin{array}{l}17 \text { partizipative Gruppendiskussionen mit } \\
\text { Krebskranken }(n=105)\end{array}$ & 2 \\
\hline EONS 2007 [22] & Europa & Onkologische Pflege & $\begin{array}{l}\text { Delphi-Studie mit EONS-Mitgliedern } \\
\text { (Runde } 1, n=165 \text { ), Funktionären (Run- } \\
\text { de } 2, n=24 \text { ) und wissenschaftlichem } \\
\text { Beirat (Runde, } 3 n=16 \text { ) }\end{array}$ & 5 \\
\hline Girgis und Butow 2009 [27] & Australien & $\begin{array}{l}\text { Cancer Survivors, Onkolo- } \\
\text { gieforschung }\end{array}$ & Overview & 2 \\
\hline Grundy und Ghazi 2009 [24] & UK & $\begin{array}{l}\text { Hämatoonkologische Pfle- } \\
\text { ge }\end{array}$ & $\begin{array}{l}\text { Literaturreview, Delphi-Studie (Runde } 1, \\
n=230, \text { Runde } 2, n=119, \text { Runde } 3, \\
n=103 \text { ) mit Royal College of Nursing } \\
\text { und Haematology and Bone Marrow } \\
\text { Transplant Forum }\end{array}$ & 3 \\
\hline Houldin et al. 2006 [30] & USA & $\begin{array}{l}\text { Cancer Survivors, onkolo- } \\
\text { gische Pflege }\end{array}$ & $\begin{array}{l}\text { ExpertInnen-Paper zu Forschungsstand } \\
\text { Cancer Survivors für Symposium, Kon- } \\
\text { sensbildung mit Symposiumsteilnehmen- } \\
\text { den }\end{array}$ & $4 a$ \\
\hline Knobf et al. 2015 [5] & USA & Onkologische Pflege & $\begin{array}{l}\text { Survey mit ONS-Mitgliedern }(n=895) \text {, } \\
\text { Literatursynthese, Konsensbildung in } \\
\text { ONS }\end{array}$ & 5 \\
\hline Murphy und Cowman 2006 [26] & Irland & Onkologische Pflege & $\begin{array}{l}\text { Fragebogenerhebung }(n=79) \text { mit Pfle- } \\
\text { genden onkologischer Spezialzentren }\end{array}$ & 5 \\
\hline Shaha et al. 2008 [2] & Schweiz & Onkologische Pflege & Systematische Literaturübersicht & 5 \\
\hline Taylor und Whayman 2009 [25] & UK & $\begin{array}{l}\text { Kolorektale onkologische } \\
\text { Pflege }\end{array}$ & $\begin{array}{l}\text { Fragebogenerhebung mit Mitgliedern } \\
\text { des kolorektalen Onkologie-Pflegenden- } \\
\text { Netzwerks }(n=25) \text {, Konsensbildung im } \\
\text { Netzwerk }\end{array}$ & 3 \\
\hline Van Cleave et al. 2016 [28] & International & Onkogeriatrische Pflege & Systematische Literaturübersicht & 3 \\
\hline
\end{tabular}

EONS European Oncology Nursing Society, ONS Oncology Nursing Society

${ }^{a}$ Level 5: Identifikation von Forschungsprioritäten der Pflege durch einen systematischen Prozess (Survey, Delphi-Studie, Metaanalyse, Fokusgruppe, systematischer Review); Level 4a: Identifikation von Forschungsprioritäten der Pflege durch Konsultation (professionelle Organisationen, Meinungen akademischer Führungskräfte, Wohlfahrtsorganisationen). Die Kriterien von Level 5 werden jedoch nicht erreicht; Level 4b: Identifikation von Forschungsprioritäten von professionellen Organisationen, die Auswirkungen auf die Pflegeforschung haben und im Prozess Level 5 erreichen; Level 3: Identifikation von Forschungsprioritäten der Pflege, erarbeitet durch kleine Interessensgruppen (diagnosebezogene oder Patientengruppen) oder durch kleine Studien, die die Kriterien von Level 4 und 5 nicht erfüllen; Level 2: Identifikation von Forschungsprioritäten, zu denen die Pflege nebst anderen Disziplinen einen Beitrag leistet, die die Kriterien von Level 5 nicht erreichen; Level 1: Identifikation von Forschungsprioritäten, zu denen die Pflege nebst anderen Disziplinen einen Beitrag leistet, die die Kriterien von Level 5 und 4 nicht erreichen (basierend auf einer Meinung) [2, 18] 
Betroffenen, Symptommanagement sowie Krebsprävention und Risikogruppen eine Rolle.

\section{Delphi-Studie}

Die Delphi-Befragung wurde von November 2013 bis Januar 2014 durchgeführt. Insgesamt wurden 32 ExpertInnen für die Studie angefragt, von denen 20 ihre schriftliche Zustimmung erteilten und 18 in der ersten, 15 in der zweiten und 13 in der dritten Befragungsrunde teilgenommen haben. Die meisten davon hatten einen Bachelor- oder Masterabschluss $(n=3)$ und/oder eine onkologische Weiterbildung $(n=8)$. Alle anderen ExpertInnen $(n=7)$ wiesen eine nachweislich große Expertise aufgrund langjähriger Berufserfahrung und/oder einer leitenden Funktion auf (Tab. 3).

In der ersten Befragungsrunde wurden bereits 34 von 40 Forschungsprioritäten mit einem eindeutigen Konsens als wichtig bewertet. Vier Ergänzungen der ExpertInnen konnten als neue Forschungsprioritäten identifiziert werden: „Bewältigungsstrategien der Betroffenen“, „Forschung zu PalliativpatientInnen“, ,Übergang vom Survivorship zur(m) RezidivpatientIn“ sowie „Versorgungsangebote für Angehörige krebskranker Menschen“. Dementsprechend wurden in der zweiten Runde 10 Forschungsprioritäten nach ihrer Wichtigkeit bewertet, von denen 5 (,Interventionen mit komplementären/alternativen Methoden“, „Interventionen mit technologiebasierten Methoden“, „Versorgungsstrukturen mit dem Fokus Survivorship“, „Übergang vom Survivorship $\operatorname{zur}(\mathrm{m})$ RezidivpatientIn“ und „Entwicklung und Testung von evidenzbasierten Standards und Leitlinien“) unter dem festgesetzten Konsenskriterium lagen. Auch in der dritten Runde konnte bei diesen 5 Forschungsprioritäten kein eindeutiger Konsens erzielt werden. Aufgrund des festgesetzten Trennwertes $\geq 51 \%$ musste jedoch lediglich

Tab. 3 Soziodemografische Merkmale der Stichprobe

\begin{tabular}{lll}
\hline Merkmal & & Anzahl $(n)$ \\
\hline Alter (min; max) & - & $29 ; 53$ \\
Geschlecht & Männlich & 2 \\
& Weiblich & 16 \\
Arbeitsort & Station & 9 \\
& Ambulanz & 1 \\
& Tagesklinik & 3 \\
& Übergreifende Bereiche & 5 \\
Berufliche Funktion & Klinische Pflege & 5 \\
& Leitende Funktion & 12 \\
Berufserfahrung & Weniger als 6 Jahre & 3 \\
Onkologie & Mehr als 6 Jahre & 15 \\
Bachelor-/ & Ja & 3 \\
Masterabschluss & Nein & 15 \\
Onkologische & Ja & 8 \\
Weiterbildung & Nein & 10 \\
\hline
\end{tabular}

die Forschungspriorität „Interventionen mit technologiebasierten Methoden“ aus der finalen Forschungsagenda ausgeschlossen werden.

Da von den ExpertInnen 4 Forschungsprioritäten mit eindeutigem Konsens ergänzt wurden und eine literaturbasierte Forschungspriorität in ihrer Wichtigkeit für den nationalen Kontext abgelehnt wurde, enthält die österreichische Forschungsagenda für onkologische Pflege in ihrer finalen Version insgesamt 43 Forschungsprioritäten zu insgesamt 9 unterschiedlichen Forschungsbereichen (Tab. 4).

\section{Diskussion}

Ziel der vorliegenden Arbeit war es, auf Basis internationaler Forschungsagenden in einem strukturierten Konsensprozess eine auf Österreich ausgerichtete Forschungsagenda für die onkologische Pflege zu entwickeln.

Hierbei wird deutlich, dass Forschungsprioritäten aus anderen Ländern im Wesentlichen auch hierzulande als wichtig beurteilt werden. Dies hängt vermutlich damit zusammen, dass die literaturgestützten Ergebnisse mehrheitlich aus europäischen Ländern stammen, in denen ähnliche Ansprüche an die onkologische Forschung bestehen.

Ähnlich wie in der Arbeit von Shaha et al. [2] befanden sich nur wenige Studien [23, 27] im unteren Bereich bezüglich ihrer Fähigkeit, Prioritäten der Pflegeforschung zu identifizieren, was darauf hindeutet, dass die literaturbasierten Forschungsprioritäten einen tatsächlichen Bedarf abbilden.

Da zusätzlich im Rahmen der Delphi-Studie Forschungsprioritäten ergänzt werden konnten, wurde sichergestellt, dass keine spezifisch nationalen Forschungsprioritäten übersehen werden. Allerdings lassen die 4 ergänzten Forschungsprioritäten nicht darauf schließen, dass diese in anderen Ländern eine untergeordnete Rolle spielen. Vor allem 2 Ergänzungen (,Bewältigungsstrategien der Betroffenen“, „Übergang vom Survivorship zur(m) RezidivpatientIn") sind in anderen Forschungsagenden möglicherweise unter allgemeiner formulierten Forschungsprioritäten subsumiert (z. B. bei der sehr allgemein formulierten Forschungspriorität ,Psychoonkologie“ von EONS [22]). Umgekehrt wurden in der Literatursynthese extrahierte Forschungsprioritäten, die als Äquivalente zu den beiden anderen Ergänzungen „Versorgungsangebote für Angehörige krebskranker Menschen“ und „Forschung zu PalliativpatientInnen“ angesehen werden können, ursprünglich unter allgemeiner formulierten Forschungsprioritäten (,Interventionen und Modelle mit dem Fokus Familie“ sowie „Interventionen für bestimmte Krankheitsphasen“) zusammengefasst, wie beispielsweise die Forschungspriorität „Unterstützung von Familienmitgliedern" von Shaha et al. [2]. Aufgrund der Bewertungen der ExpertInnen wurde jedoch beschlossen, 
Tab. 4 Österreichische Forschungsagenda für onkologische Pflege

\begin{tabular}{|c|c|c|c|}
\hline Forschungsbereiche & Forschungsprioritäten & ÜS \% $(N)$ & $M(S D)$ \\
\hline \multirow{6}{*}{$\begin{array}{l}\text { Krankheits- und } \\
\text { Therapieerfahrungen der } \\
\text { PatientInnen }\end{array}$} & Untersuchung der Bewältigungsstrategien von Betroffenen & $100(13)^{\mathrm{b}}$ & $1,15( \pm 0,38)^{\mathrm{b}}$ \\
\hline & Untersuchung der Symptombelastung & $100(18)^{\mathrm{a}}$ & $1,17( \pm 0,38)^{\mathrm{a}}$ \\
\hline & Untersuchung krankheits- und therapiebedingter Bedürfnisse & $100(18)^{\mathrm{a}}$ & $1,22( \pm 0,43)^{\mathrm{a}}$ \\
\hline & Untersuchung psychosozialer Auswirkungen & $94,4(18)^{\mathrm{a}}$ & $1,50( \pm 0,62)^{\mathrm{a}}$ \\
\hline & Untersuchung der Krankheitserfahrung aus Sicht der Betroffenen & $100(18)^{\mathrm{a}}$ & $1,56( \pm 0,51)^{\mathrm{a}}$ \\
\hline & Untersuchung von Symptomclustern & $92,3(18)^{b}$ & $1,69( \pm 0,63)^{b}$ \\
\hline \multirow{3}{*}{$\begin{array}{l}\text { Entwicklung von } \\
\text { Assessmentinstrumenten zu } \\
\text { Erkennung und Beurteilung }\end{array}$} & Assessment des Symptommanagements & $94,4(18)^{\mathrm{a}}$ & $1,22( \pm 0,55)^{\mathrm{a}}$ \\
\hline & $\begin{array}{l}\text { Assessment von psychosozialen Risiken von Menschen mit Krebser- } \\
\text { krankungen }\end{array}$ & $100(18)^{\mathrm{a}}$ & $1,50( \pm 0,51)^{\mathrm{a}}$ \\
\hline & $\begin{array}{l}\text { Assessmentinstrumente für spezielle PatientInnengruppen (z. B. Pallia- } \\
\text { tivpatientInnen, onkogeriatrische PatientInnen) }\end{array}$ & $94,4(18)^{\mathrm{a}}$ & $1,72( \pm 0,58)^{\mathrm{a}}$ \\
\hline \multirow{5}{*}{$\begin{array}{l}\text { Entwicklung und Testung von } \\
\text { Interventionen }\end{array}$} & Interventionen zum Symptommanagement & $100(18)^{\mathrm{a}}$ & $1,11( \pm 0,32)^{\mathrm{a}}$ \\
\hline & Programme zur Krebsprävention & $100(16)^{\mathrm{a}}$ & $1,25( \pm 0,45)^{\mathrm{a}}$ \\
\hline & Interventionen zu spezifischen pflegesensitiven Outcomes & $100(18)^{\mathrm{a}}$ & $1,28( \pm 0,46)^{\mathrm{a}}$ \\
\hline & $\begin{array}{l}\text { Interventionen zu bestimmten Krankheitsphasen (z. B. Diagnose, Sur- } \\
\text { vivorship) }\end{array}$ & $100(18)$ & $1,56( \pm 0,51)$ \\
\hline & Interventionen mit komplementären/alternativen Methoden & $76,9(13)^{\mathrm{c}}$ & $2,23( \pm 0,44)^{\mathrm{c}}$ \\
\hline \multirow[t]{3}{*}{ PatientInnenedukation } & $\begin{array}{l}\text { Identifikation von Informations-, Beratungs- und Schulungsbedürfnis- } \\
\text { sen }\end{array}$ & $100(18)^{\mathrm{a}}$ & $1,28( \pm 0,46)^{\mathrm{a}}$ \\
\hline & $\begin{array}{l}\text { Entwicklung und Evaluation von Programmen zu Information, Bera- } \\
\text { tung und Schulung von KrebspatientInnen }\end{array}$ & $100(18)^{\mathrm{a}}$ & $1,28( \pm 0,46)^{\mathrm{a}}$ \\
\hline & $\begin{array}{l}\text { Entwicklung und Evaluation von Programmen zu Information, Bera- } \\
\text { tung und Schulung im Bereich Krebsprävention }\end{array}$ & $94,4(18)^{\mathrm{a}}$ & $1,28( \pm 0,56)^{\mathrm{a}}$ \\
\hline \multirow{5}{*}{$\begin{array}{l}\text { Spezifische } \\
\text { PatientInnengruppen }\end{array}$} & Forschung zu benachteiligten PatientInnengruppen & $100(18)^{\mathrm{a}}$ & $1,39( \pm 0,50)^{\mathrm{a}}$ \\
\hline & Forschung zu PalliativpatientInnen & $100(15)^{\mathrm{b}}$ & $1,40( \pm 0,51)^{\mathrm{b}}$ \\
\hline & Forschung zu onkogeriatrischen PatientInnen & $94,4(18)^{\mathrm{a}}$ & $1,44( \pm 0,62)^{\mathrm{a}}$ \\
\hline & Forschung zu Krebsrisikogruppen & $100(16)^{\mathrm{a}}$ & $1,56( \pm 0,51)^{\mathrm{a}}$ \\
\hline & Forschung zu PatientInnen mit seltenen Krebserkrankungen & $94,4(18)^{\mathrm{a}}$ & $1,72( \pm 0,58)^{\mathrm{a}}$ \\
\hline \multirow{6}{*}{$\begin{array}{l}\text { Cancer Survivors und } \\
\text { Survivorship }\end{array}$} & Information, Beratung und Schulung für Cancer Survivors & $94,4(18)^{\mathrm{a}}$ & $1,33( \pm 0,59)^{\mathrm{a}}$ \\
\hline & Bedeutung des Survivorship für Betroffene & $100(18)^{\mathrm{a}}$ & $1,39( \pm 0,50)^{\mathrm{a}}$ \\
\hline & Bedürfnisse von Cancer Survivors & $100(18)^{\mathrm{a}}$ & $1,50( \pm 0,51)^{\mathrm{a}}$ \\
\hline & Interventionen für Cancer Survivors & $94,4(18)^{\mathrm{a}}$ & $1,56( \pm 0,62)^{\mathrm{a}}$ \\
\hline & Übergang vom Survivorship zur(m) RezidivpatientIn & $69,2(13)^{\mathrm{c}}$ & $2,31( \pm 0,48)^{\mathrm{c}}$ \\
\hline & Versorgungsstrukturen mit dem Fokus Survivorship & $53,8(13)^{\mathrm{c}}$ & $2,46( \pm 0,52)^{\mathrm{c}}$ \\
\hline \multirow{6}{*}{$\begin{array}{l}\text { Angehörige/Familien von } \\
\text { Menschen mit } \\
\text { Krebserkrankungen }\end{array}$} & $\begin{array}{l}\text { Information, Beratung und Schulung für betroffene Angehörige bzw. } \\
\text { Familien }\end{array}$ & $100(18)^{\mathrm{a}}$ & $1,22( \pm 0,43)^{\mathrm{a}}$ \\
\hline & Bedürfnisse von betroffenen Angehörigen bzw. Familien & $100(18)^{\mathrm{a}}$ & $1,33( \pm 0,49)^{\mathrm{a}}$ \\
\hline & $\begin{array}{l}\text { Erfahrungen und Auswirkungen der bzw. auf betroffene(n) Angehöri- } \\
\text { ge(n)/Familien }\end{array}$ & $100(16)^{\mathrm{a}}$ & $1,44( \pm 0,51)^{\mathrm{a}}$ \\
\hline & Versorgungsangebote für Angehörige krebskranker Menschen & $100(15)^{\mathrm{b}}$ & $1,53( \pm 0,52)^{b}$ \\
\hline & $\begin{array}{l}\text { Entwicklung von Assessmentinstrumenten für betroffene Angehörige } \\
\text { bzw. Familien (z. B. psychosoziales Risikoassessment) }\end{array}$ & $100(18)^{\mathrm{a}}$ & $1,61( \pm 0,50)^{\mathrm{a}}$ \\
\hline & Interventionen und Modelle mit dem Fokus Familie & $94,4(18)^{\mathrm{a}}$ & $1,67( \pm 0,59)^{\mathrm{a}}$ \\
\hline \multirow[t]{4}{*}{ Ethische und rechtliche Aspekte } & $\begin{array}{l}\text { Ethische Aspekte in der Kommunikation zwischen Pflegenden und } \\
\text { PatientInnen }\end{array}$ & $100(18)^{\mathrm{a}}$ & $1,33( \pm 0,49)^{\mathrm{a}}$ \\
\hline & $\begin{array}{l}\text { Konzepte zur Entscheidungsfindung (z. B. Information und Einwilli- } \\
\text { gung bei Therapie- und Behandlungsverfahren) }\end{array}$ & $94,4(18)^{\mathrm{a}}$ & $1,39( \pm 0,61)^{\mathrm{a}}$ \\
\hline & PatientInnen-Sicherheit im Krankenhaus (z. B. Medikationsfehler) & $94,4(18)^{\mathrm{a}}$ & $1,39( \pm 0,61)^{\mathrm{a}}$ \\
\hline & $\begin{array}{l}\text { Gerechte Verteilung von bzw. gerechter Zugang zu spezifischer onko- } \\
\text { logischer Betreuung (z. B. Palliativpflegeplatz) }\end{array}$ & $93,3(15)^{\mathrm{b}}$ & $1,47( \pm 0,64)^{\mathrm{b}}$ \\
\hline
\end{tabular}


Tab. 4 Österreichische Forschungsagenda für onkologische Pflege (Fortsetzung)

\begin{tabular}{|c|c|c|c|}
\hline Forschungsbereiche & Forschungsprioritäten & ÜS \% $(N)$ & $M(S D)$ \\
\hline \multirow{5}{*}{$\begin{array}{l}\text { Gesundheitswesen und } \\
\text { Entwicklung der onkologischen } \\
\text { Pflege }\end{array}$} & $\begin{array}{l}\text { Entwicklung und Klärung von zukünftigen Rollen von Pflegenden in } \\
\text { der Onkologie (z. B. Advanced Practice Nurse) }\end{array}$ & $94,4(18)^{a}$ & $1,22( \pm 0,55)^{\mathrm{a}}$ \\
\hline & $\begin{array}{l}\text { Versorgungsangebote für KrebspatientInnen betreffend onkologische } \\
\text { Einrichtungen und Dienstleistungen }\end{array}$ & $100(18)^{\mathrm{a}}$ & $1,44( \pm 0,51)^{\mathrm{a}}$ \\
\hline & $\begin{array}{l}\text { Forschung zur Implementierung wissenschaftlicher Erkenntnisse in die } \\
\text { Pflegepraxis }\end{array}$ & $94,4(18)^{\mathrm{a}}$ & $1,50( \pm 0,79)^{\mathrm{a}}$ \\
\hline & Case- und Entlassungsmanagement & $94,1(18)^{\mathrm{a}}$ & $1,59( \pm 0,62)^{\mathrm{a}}$ \\
\hline & $\begin{array}{l}\text { Entwicklung und Testung von evidenzbasierten Standards und Leitlini- } \\
\text { en }\end{array}$ & $76,9(13)^{\mathrm{c}}$ & $2,23( \pm 0,44)^{\mathrm{c}}$ \\
\hline
\end{tabular}

$\ddot{U} S$ Übereinstimmungsgrad, $M$ Mittelwert bezüglich der Wichtigkeit - niedrige Werte weisen auf eine höhere Wichtigkeit hin,

$S D$ Standardabweichung

${ }^{a}$ Werte aus Befragungsrunde 1

${ }^{b}$ Werte aus Befragungsrunde 2

${ }^{\mathrm{c}}$ Werte aus Befragungsrunde 3

die Ergänzungen explizit als österreichische Forschungsprioritäten aufzunehmen.

Der Ausschluss von ,Interventionen mit technologiebasierten Methoden" durch die ExpertInnen ist insofern interessant, weil es sich gerade hier in Form von eSmart und Telenursing um einen aufstrebenden Bereich handelt, in dem zunehmend sowohl international [31] als auch im deutschsprachigen Raum [32] geforscht wird. Allerdings wurde in etwa die Hälfte der Befragten aus einem großen, urbanen, technologisch gut ausgestatteten Universitätsspital rekrutiert. Aus diesem Grund würde möglicherweise ein geringer Forschungsbedarf für Interventionen mit technologiebasierten Methoden angegeben. Wenn man jedoch davon ausgeht, dass technologiebasierte Methoden auch in Gebieten mit feinmaschiger Gesundheitsversorgung für das Selbstmanagement der PatientInnen [31] genutzt werden können, dürfte diesem Bereich auch in Österreich künftig größere Bedeutung beigemessen werden.

\section{Limitationen}

Da in Österreich keine ExpertInnen zur Verfügung stehen, die direkt in der onkologischen Pflegeforschung tätig sind und Onkologiepflegende mit akademischer Ausbildung hierzulande ebenfalls sehr selten sind, mussten die Einschlusskriterien für die Delphi-Studie weit gefasst werden. Folglich wurden die Forschungsprioritäten in Hinblick auf deren Wichtigkeit für den österreichischen Forschungskontext von einer Gruppe bewertet, der es möglicherweise an umfassender wissenschaftlicher Expertise fehlte. Hier wurde jedoch darauf geachtet, dass die Heterogenität der ExpertInnen insofern erhöht wird, als diese aus einem Universitätsspital als auch einer landesweit tätigen Institution mit VertreterInnen aus unterschiedlichsten Settings (stationär bis ambulant) rekrutiert wurden, wenngleich es sich im zweiten Fall ausschließlich um Pflegende aus dem hämatoonkologischen Bereich handelte. Darüber hinaus ent- sprachen nur 32 Personen den festgesetzten Kriterien, von denen 13 ExpertInnen an der letzten Befragungsrunde teilgenommen haben. Allerdings stellt bei der Delphi-Methode die Stichprobengröße ein weniger wichtiges Kriterium dar, als der ExpertInnenstatus [20]. Da nur eine literaturgestützt identifizierte Forschungspriorität mit eindeutigem Konsens aus der Forschungsagenda ausgeschlossen werden musste, könnten folglich Forschungsprioritäten in die Forschungsagenda eingeflossen sein, die von wissenschaftlichen ExpertInnen möglicherweise als nachrangig eingestuft worden wären. Zudem könnte aufgrund von fehlenden Werten der Konsens bei 9 von insgesamt 44 bewerteten Items überoder unterschätzt worden sein.

\section{Implikationen und Schlussfolgerungen}

Die Forschungsagenda bildet erstmalig einen Rahmen für die österreichische Forschung auf dem Gebiet der onkologischen Pflege, mit der diese umfassender geplant und strategisch ausgerichtet werden kann. Sie enthält Forschungsprioritäten, die einen wichtigen Beitrag zu einer verbesserten, evidenzbasierten Pflegepraxis leisten können und die ein breites Spektrum onkologischer Pflegeforschung umfassen. Von präventiven pflegerischen Maßnahmen über akute Krankheitsphasen bis hin zum Survivorship und der Palliativpflege sind auch Angehörige, psychosoziale Aspekte sowie die Entwicklung der onkologischen Pflege im Gesundheitswesen erfasst.

Allerdings ist die Forschungsagenda aufgrund der oben angeführten Limitationen als erster wichtiger Schritt anzusehen. Sie muss überarbeitet und ergänzt werden, sobald in Österreich ExpertInnen aus der onkologischen Pflegeforschung zur Verfügung stehen. Ferner wird empfohlen, weiterführende Arbeiten an der Forschungsagenda aus PatientInnen-, Angehörigen- und interdisziplinärer Sichtweise $\mathrm{zu}$ validieren und in einen weiter gefassten, nationalen Kontext durch eine Zusammenarbeit mit anderen For- 
schungsinstitutionen, inklusive einer offiziellen berufspolitischen Verlautbarung, einzubetten.

Zusammenfassend kann konstatiert werden, dass die Forschungsagenda einen ersten wichtiger Schritt darstellt, mit dem eine umfassende Prioritätensammlung entwickelt werden konnte, dem jedoch weitere Schritte folgen müssen, um eine Forschungsagenda mit nationaler Gültigkeit und hoher Repräsentativität der Forschungsbedarfe in Österreich zur Verfügung stellen zu können.

Acknowledgements Open access funding provided by University of Vienna.

Open Access Dieser Artikel wird unter der Creative Commons Namensnennung 4.0 International Lizenz (http://creativecommons.org/ licenses/by/4.0/deed.de) veröffentlicht, welche die Nutzung, Vervielfältigung, Bearbeitung, Verbreitung und Wiedergabe in jeglichem Medium und Format erlaubt, sofern Sie den/die ursprünglichen Autor(en) und die Quelle ordnungsgemäß nennen, einen Link zur Creative Commons Lizenz beifügen und angeben, ob Änderungen vorgenommen wurden.

\section{Literatur}

1. Imhof, L., Abderhalden, C., Cignacco, E., Eicher, M., Mahrer-Imhof, R., Schubert, M., et al. (2008). Swiss Research Agenda for Nursing (SRAN): Die Entwicklung einer Agenda fur die klinische Pflegeforschung in der Schweiz. Pflege, 21, 375-384.

2. Shaha, M., Schmid-Büchi, S., Abt, J., Mathis-Jäggi, F., Holdener, E., Riederer, E., et al. (2008). Der Beitrag der Onkologiepflege zur Swiss Research Agenda for Nursing - SRAN. Pflege, 21, 385-403.

3. Jahn, P., \& Pflegeforschung Deutschland, L. M. (2013). Aktueller Stand und Perspektiven. Forum, 28, 111-114.

4. McIlfatrick, S. J., \& Keeney, S. (2003). Identifying cancer nursing research priorities using the Delphi technique. J Adv Nurs, 42, 629-636.

5. Knobf, M.T., Cooley, M.E., Duffy, S., Doorenbos, A., Eaton, L., Given, B., et al. (2015). The 2014-2018 oncology nursing society research agenda. Oncol Nurs Forum, 42, 450-465.

6. American Cancer Society (2015). Cancer facts \& figures 2015. http://www.cancer.org/Research/CancerFactsStatistics/cancerfacts figures2015/cancer-facts-and-figures-2015. Zugegriffen: 31. Aug. 2016.

7. Statistik Austria (2014). Krebsinzidenz und Krebsmortalität in Österreich. Statistik Austria. http://www.statistik.at/web_de/statisti ken/gesundheit/krebserkrankungen/index.html. Zugegriffen: 22 . Apr. 2015.

8. Bachmann-Mettler, I., \& Eicher, M. (2011). Nationales Krebsprogramm für die Schweiz. Oncosuisse. http://www.oncosuisse.ch/ file/oncosuisse/nkp/2011-2015/kurzversion/NKP_Kurzversion_d. pdf. Zugegriffen: 31. Aug. 2016.

9. Preyde, M., \& Synnott, E. (2009). Psychosocial intervention for adults with cancer: A meta-analysis. J Evid Based Soc Work, 6, 321-347.

10. Gao, W. J., \& Yuan, C.R. (2011). Self-management programme for cancer patients: A literature review. Int Nurs Rev, 58, 288-295.

11. Matzka, M., Mayer, H., Kock-Hodi, S., Moses-Passini, C., Dubey, C., Jahn, P., et al. (2016). Relationship between resilience, psychological distress and physical activity in cancer patients: A crosssectional observation study. PloS one, 11, e0154496.

12. Kobleder, A., Raphaelis, S., \& Senn, B. (2014). Symptommanagement bei vulvären Neoplasien. Status quo und künftige Entwicklungen. Onkologiepflege, 3, 9-11.
13. Grössmann, N., Wild, C., \& Onkologika, M. J. (2016). Übersicht zu Nutzenbewertungen und Refundierungspolitiken in Europa. Rapid Assessment LBI-HTA Nr. 08.2016. http://eprints.hta.lbg.ac.at/ 1091/1/Rapid_Assessment_008.pdf. Zugegriffen: 20. Juni 2017.

14. Klastersky, J. A. (2014). Adverse events of targeted therapies. Curr Opin Oncol, 26, 395-402.

15. Bundesministerium für Gesundheit (2012). Österreichischer Struktruplan Gesundheit 2012 inklusive Großgeräteplan. https://www. bmgf.gv.at/cms/home/attachments/1/0/1/CH1071/CMS113698338 2893/oesg_2012_text_ohne_matrizen_-_neue_links.pdf. Zugegriffen: 20. Juni 2017.

16. Bundesministerium für Gesundheit (2014). Krebsrahmenprogramm Österreich. https://extranet.who.int/ncdccs/Data/AUT_B5_ krebsrahmenprogramm.pdf. Zugegriffen: 20. Juni 2017.

17. Jönsson, B., Hofmarcher, T., Lindgren, P., \& Wilking, N. (2016). Comparator report on patient access to cancer medicines in europe revisited. IHE report. http://lup.lub.lu.se/search/ws/files/11713673/ IHE_Report_2016_4_.pdf. Zugegriffen: 20. Juni 2017.

18. Ross, F., Smith, E., Mackenzie, A., \& Masterson, A. (2004). Identifying research priorities in nursing and midwifery service delivery and organisation: A scoping study. Int Journal Nurs Stud, 41, 547-558.

19. Burnette, D., Morrow-Howell, N., \& Chen, L. M. (2003). Setting priorities for gerontological social work research: A national Delphi study. Gerontologist, 43, 828-838.

20. Keeney, S., McKenna, H., \& Hasson, F. (2010). The Delphi technique in nursing and health research. Oxford: Wiley-Blackwell.

21. Schafer, J. L., \& Graham, J.W. (2002). Missing data: Our view of the state of the art. Psychol Methods, 7, 147-177.

22. EONS (2011). Modified Delphi survey establishes EONS research agenda until. Eur J Oncol Nurs, 2007(11), 1-3.

23. Corner, J., Wright, D., Hopkinson, J., Gunaratnam, Y., McDonald, J. W., \& Foster, C. (2007). The research priorities of patients attending UK cancer treatment centres: Findings from a modified nominal group study. Br J Cancer, 96, 875-881.

24. Grundy, M., \& Ghazi, F. (2009). Research priorities in haematooncology nursing: Results of a literature review and a Delphi study. Eur J Oncol Nurs, 13, 235-249.

25. Taylor, C., \& Whayman, K. (2009). Raising the profile of the National Colorectal Cancer Nurses Network: an email survey. Gastrointenstinal Nursing, 7, 24-30.

26. Murphy, A., \& Cowman, S. (2006). Research priorities of oncology nurses in the Republic of Ireland. Cancer Nurs, 29, 283-290.

27. Girgis, A., \& Butow, P. (2009). Cancer survivorship: Research priorities at the national and international levels. CancerForum, 33, 194-197.

28. Van Cleave, J.H., Kenis, C., Sattar, S., Jabloo, V.G., Ayala, A.P., $\&$ Puts, M. (2016). A research agenda for Gero-oncology nursing. Semin Oncol Nurs, 32, 55-64.

29. Berger, A. M., Cochrane, B., \& Mitchell, S. A. (2009). The 20092013 research agenda for oncology nursing. Oncol Nurs Forum, 36, E274-E282.

30. Houldin, A., Curtiss, C. P., \& Haylock, P. J. (2006). Executive summary: The state of the science on nursing approaches to managing late and long-term sequelae of cancer and cancer treatment. American J Nurs, 106, 54-59.

31. Kearney, N., McCann, L., Norrie, J., Taylor, L., Gray, P., McGeeLennon, M., et al. (2009). Evaluation of a mobile phone-based, advanced symptom management system (ASyMS) in the management of chemotherapy-related toxicity. Support Care Cancer, 17, 437-444.

32. Dubey, C., Hoeppli, C., Carrino, F., Mugellini, E., Kueng, M., Kropf, S., et al. (2014). Enhancing symptom self-management through a smartphone application: Developmentand pilot testing. Support Care Cancer, 1, 225. 